\title{
THE ROLE OF INTESTINAL MICROBIOTA IN THE PATHOGENESIS OF METABOLIC DISEASES
}

\author{
Iwona Węgielska, Joanna Suliburska ${ }^{\bowtie}$ \\ Department of Human Nutrition and Hygiene, Poznań University of Life Sciences \\ Wojska Polskiego 31, 60-624 Poznań, Poland
}

\begin{abstract}
The incidence of metabolic diseases is increasing rapidly all over the world. This situation has led researchers to attempt to explain the pathomechanisms of these disorders and to develop specific recommendations for the prevention and treatment of diseases such as obesity, type- 2 diabetes, and atherosclerosis. Recent studies show clear evidence of the role of human intestinal microbiota in health and in predispositions to diseases. Gut microbiota affect a number of complex metabolic reactions, significantly altering the functioning of the human body. Numerous experiments have shown the key role played by the formation process of the intestinal ecosystem in the early stages of human life for programming its metabolic health. The following article is a compilation of the literature available on the formation of the complex intestinal ecosystem and its impact on the incidence of diseases such as obesity, type-2 diabetes, and atherosclerosis.
\end{abstract}

Key words: intestinal microbiota, metabolic diseases, obesity, diabetes mellitus, atherosclerosis

\begin{abstract}
Abbreviations: $\mathrm{HMO}$ - human milk oligosaccharides, GALT - gut-associated lymphatic tissue, MALT lymphoid tissue associated with mucosal membranes, ChREBP - carbohydrate response element-binding protein, SREBP-1 - liver sterol response elementbinding protein type-1, FIAF fasting-induced adipocyte factor, PPAR - peroxisome proliferator-activated receptor coactivator $\gamma$, LPS - lipopolysaccharide, eCB - endocannabinoid system, FXR - farnesoid X nuclear receptor, GPCR - G protein-coupled receptor, VSG vertical sleeve gasterectomy, TMA - trimethylamine, TMAO - trimethylamine oxide, SRA - scavenger receptor A, PCA - protocatechuic acid, Cy-3-G - cyanidin glucoside, LXR - liver X receptor.
\end{abstract}

\section{INTRODUCTION}

The human gut forms a rich bacterial ecosystem. Depending on the individual, intestinal microbiota may consist of up to 1000 different species of microorganisms. Recent research in the field of molecular biology has provided information on the size of the genome of intestinal bacteria (Dietert and Dietert, 2015). Microbiota genes exceed host genes in number by a factor of a hundred, and are thus an important source of numerous enzymes and provide a variety of metabolic pathways while regulating the immune system. In the literature, intestinal microbiota is often referred to as the "bacterial organ" (Turnbaugh et al., 2009). This emphasizes the number of functions of the microbiota, its large mass ( 1.5 to $2 \mathrm{~kg}$ ), and its plurality of functional connections with numerous internal organs (Clark et al., 2012; Turnbaugh et al., 2009).

Research in recent years has suggested that the condition of the intestinal microbiota plays a significant role in the pathogenesis of obesity, atherosclerosis, type- 2 diabetes, and insulin resistance. The rapidly growing problem of metabolic disorders associated with obesity cannot be explained by genetic and epigenetic factors and environmental conditions 
alone. The analysis of calorie balance in the diet of the population is also insufficient to explain the scale of this harmful phenomenon. However, new light has been shed on the problem by research into prebiotics, probiotics, and antibacterial agents. Currently, there is no doubt that the intestinal microbiota plays an important role in the pathogenesis of metabolic disorders, but further studies need to be able to fully utilize the accumulated knowledge for the practical treatment of patients. The following article is an attempt to compile the most important information currently available on the evolution of the bacterial intestinal ecosystem and its role in the development of metabolic diseases.

\section{ESTABLISHMENT OF THE HUMAN MICROBIOTA ECOSYSTEM}

In order to fully understand the role of intestinal microbiota in the functioning of the host metabolism, it is important to pay attention to the development process of the infant gut, which runs parallel to, but is to a large extent dependent on, the process of intestinal colonization by specific bacterial strains. Data from the literature clearly shows that those early stages of intestinal development involving the formation of the bacterial ecosystem are critical in shaping the host's metabolism (Arrieta et al., 2014; Clark et al., 2012). They constitute a "critical window" for programming human metabolic health and its metabolic phenotype shape. Disorders of the intestinal progress in the early stages can result in far-reaching metabolic dysfunctions manifesting themselves in childhood and adulthood (Arrieta et al., 2014; Clark et al., 2012). The question of the sterility of a newborn child's gastrointestinal tract continues to be debated. Some studies have shown that in the majority of newborns, the gastrointestinal tract at birth is sterile (Arrieta et al., 2014; Clark et al., 2012). However, it has been noted that in the case of some children participating in the studies, colonization of the gut occurred in utero through the upward pathway from the genital and lower gastrointestinal tract of the mother. Analysis has showed that among those infants born prematurely, the degree of intestinal colonization was significantly lower than in those born after 273 days of gestation. It is clear that for preterm infants, colonization progresses mainly in the direction of pathogenic strains, while in children born in term, E. coli gradually supplants potentially dangerous strains, mainly Klebsiella pneumoniae. E. coli is predominant in newborns precolonized in utero, and its presence prevents the invasion of gramnegative pathogenic strains, although $E$. coli is considered an opportunistic organism.

It is indisputable that the process of intestinal colonization proceeds at great pace in the first days of life. At the same time, the intestines of newborn babies go through important stages of development, depending largely on bacterial colonization (Clark et al., 2012). Extensive research involving infants has shown that the gut microbiota is highly unstable in the first three years of life (Galdeano and Perdigo, 2006; Lozupone et al., 2013). It is much less taxonomically diverse than in adults, but more bacterially diverse (Lozupone et al., 2013). There are differences in the rate of colonization of the gastrointestinal tract between preterm newborns and those born at term; however, in each case, a delay in the process increases the risk of colonization by pathogenic strains. The upper part of the gastrointestinal tract is colonized by the bacteria that normally inhabit the oral cavity, whereas the lower part develops flora characteristic of the lower gastrointestinal tract of the mother. In the first days of life, the intestinal environment is aerobic and supports facultative anaerobic bacteria, mainly Enterobacteriaceae. A few days later, the intestine becomes an anaerobic environment, enabling the existence of obligate anaerobic bacteria (Bifidobacterium, Clostridium, Bactericides). During the first weeks of life, the composition of the child's intestinal microbiota is reminiscent of the microbiota of the skin and genital tract of the mother (Enterococcaceae, Streptococcaceae, Lactobacillaceae Clostridiaceae, Bifidobacteriaceae). The initial months of human life are a time when the only food is milk, which affects the composition of intestinal microbiota. The intestinal environment is dominated by oligosaccharide-fermenting bacteria, whose food source is milk (Bifidobacterium) (Galdeano and Perdigo, 2006). The introduction of new, solid foods to the baby's diet is the next stage in the development of the intestinal ecosystem. The supply of different nutrients, including polysaccharides generally indigestible by human enzymes, results in the growth of the bacterial population of the Bacteroides, Clostridium, 
Ruminococcus families, with a simultaneous decline in the population of Bifidobacterium and Enterobacteriaceae (Fallani et al., 2011). Between 12 and 30 months, an infant's intestinal bacterial ecosystem in composition resembles that present in adults, being dominated by bacteria of the genera Ruminococcaceae, Lachnospiraceae, Bacteroidaceae, and Prevotellaceae (Galdeano and Perdigo, 2006).

Proper microbial colonization induces the anatomical development of the intestine by affecting the structure of the microvilli and by increasing the epithelium generative capacity. Symbiotic flora is very important in shaping the gut-associated lymphatic tissue (GALT) (Koren et al., 2012), which plays the crucial role in the immunological activity of the whole system of lymphoid tissue associated with mucosal membranes (MALT). It consists of more than $75 \%$ of all lymphatic cells in the immune system. In the body, about $80 \%$ of all immunoglobulin is produced in the intestine (Koren et al., 2012). GALT produces IgA antibodies, referred to as secretory IgA (S-IgA - secretory $\operatorname{Ig} \mathrm{A}$ ), which are secreted at the mucosal surface and are responsible for capturing antigens and preventing their passage through the mucosal barrier into the body (Koren et al., 2012). GALT system's specific structure makes it the locus of the immune response; specialized cells operating within it are responsible, through differentiation and the secretion of cytokines, for inducing inflammation or tolerance towards antigens (development of food tolerance; Koret et al., 2012). The role of symbiotic microflora in the development of the infant immune system should be emphasized, because the common denominator for obesity and metabolic dysfunction is a generalized mild inflammation with its source in the immune system (Clark et al., 2012).

\section{SELECTED FACTORS SIGNIFICANTLY AFFECTING THE COMPOSITION OF HUMAN INTESTINAL MICROBIOTA IN THE EARLY STAGES OF LIFE}

In order to identify the factors that affect the homeostasis of the intestinal microflora, and thus the development of metabolic dysfunctions associated with it, we compiled the most significant prenatal, perinatal, and postnatal events that affect normal microflora composition.

\section{THE PRENATAL PERIOD}

The initial bacteria for the development of infants' intestinal ecosystem are received from the mother during the prenatal and perinatal periods (Clark et al., 2012). According to the literature, and contrary to earlier beliefs, amniotic fluid, meconium, placenta, and fetal membranes are not sterile, and the microbiota present in them has an impact beyond pregnancy and childbirth (Koleva et al., 2015). In the amniotic fluid and placenta of women with normal, full-term pregnancies (discounting medical conditions), types of bacteria normally present in the oral cavity have been identified (Firmicutes, Actinobacteria, bacteroidetes, Fusobacteria, Proteobacteria; Koleva et al., 2015). Current research shows that meconium is not sterile, which suggests the possibility that microorganisms located in the amniotic fluid are transmitted to the unborn fetus (Koleva et al., 2015). Despite the lack of conclusive evidence, it has been suggested that the placenta mediates the transfer of maternal micro-organisms to the fetus (Koren et al., 2012). A comparison of the microbiota present in the meconium with that of the amniotic fluid, vagina, and mouth shows that the meconium and amniotic fluid have the most similar composition of microorganisms (Mshvildadze et al., 2010). These results also show the microbial similarity between the meconium and adult intestines (Koleva et al., 2015). In healthy, full-term infants, bacteria belonging to the genera Enterobacteriaceae (including Escherichia coli and Shigella sp.), Streptococci, Enterococci, Staphylococci (including Staphylococcus epidermidis), and Bifidobacteria have been identified (Koleva et al., 2015). These findings suggest that abnormal microbiota composition in the body of a pregnant woman may be transferred to the unborn fetus, with all the consequences, including immune and metabolic syndrome. Some of the factors that contribute to dysbiosis of the maternal organism include infection of the genital tract, antibiotic treatments (especially broad spectrum antibiotics), and a poor diet rich in fats and sugars (Koleva et al., 2015).

Antibiotic treatment is a key factor that influences infant gut microbiota as early as the prenatal period. A seven-year study carried out in USA has shown that children at risk of exposure to antibiotics during the second or third trimesters were characterized by 
a $84 \%$ risk of childhood obesity (Koren et al., 2012). In addition, as indicated by other observations, prenatal exposure to antibiotics results in lower birth weight and methylation of imprinted genes. Moreover, the reduction in the birth weight of newborns was associated with an excessive growth of body fat, the development of childhood obesity, and related metabolic complications due to intestinal dysbiosis (Koren et al., 2012). Similar observations were provided by a study involving Danish women, where an increased risk of asthma was observed among the children of mothers treated many times with antibiotics before, during, and after pregnancy (Ridaura et al., 2013). It may be concluded that prenatal exposure to antibiotics has a direct impact on the composition and/or function of the intestinal microbiota and the physiology of the infant after birth. Pathologically modified microbiota affected the development of the intestinal immune system in a specific way by programming it to tend towards chronic mild inflammation, which in the light of present research seems to be the common denominator of immune-mediated diseases and metabolic syndrome (Blaser and Falkow, 2009).

\section{THE PERINATAL PERIOD}

Birth is an important stage in the development of the intestinal microbiota of the human body. Recent years have seen a significant increase in the proportion of pregnancies concluded by Cesarean section around the world (Tsakok et al., 2013). However, it should be emphasized that Cesarean section is not a natural way of giving birth and adversely affects the type and number of bacteria acquired by the newborn (Arrieta et al., 2014). A recent study carried out in the USA showed a $46 \%$ increase in the risk of obesity in children born by Cesarean section compared to those born through natural childbirth. During vaginal birth, the infant is in contact with the vaginal microbiota and the gastrointestinal tract, and in the first few days of its life, Lactobacillus and Bifidobacterium bacilli become dominant flora in the gastrointestinal tract (Koren et al., 2012). These are accompanied by microorganisms from the genera Bacteroides and Clostridium (Eubacterium). Cesarean section prevents the infant from coming into contact with the vaginal microbiota and mother's gastrointestinal tract. Colonization of the gastrointestinal tract of the newborn then occurs primarily from the hospital environment and the mother's skin bacteria. Babies born by Cesarean section have a reduced number of obligate anaerobic bacteria of the genus Bifidobacterium and the species Bacteroides fragilis than those born naturally, and the process of colonization falls behind schedule (Cox and Blaser, 2013). Observations of the bacterial ecosystem of children born by Cesarean section at 24 months showed significantly lower diversity of species than their peers born through vaginal birth. There is also a lack of intestinal colonization by bacteria of the genus Bacteroides in first year of age (Cox and Blaser, 2013). The intestinal ecosystem is then reminiscent of the bacteria that reside on the skin, and is rich mainly in bacteria of the genera Staphylococcus, Corynebacterium, Propionibacterium, and Clostridium (Hooper et al., 2012).

As mentioned above, Cesarean birth and the disbiosis of bacterial colonization of the infant gut associated with it result in the development of immune and metabolic disorders later in childhood and in adult life (Koren et al., 2012). The results of several studies have confirmed the association between Cesarean section and obesity in children, and this relationship has been found to be stronger than the relation between maternal and child obesity (Penders et al., 2006).

\section{THE POSTNATAL PERIOD}

After birth, the means of feeding seems to be the main factor in the continued development of the newborn's intestinal microbiota. The superiority of breastfeeding in terms of the proper formation of qualitative and quantitative composition of intestinal ecosystem should be emphasized. Breast milk is referred as a "natural symbiotic". Breast milk contains beneficial probiotic bacteria and the oligosaccharides that stimulate their intestinal colonization (Jeurink et al., 2013). Studies of breast-fed children show a rapid increase in the number of intestinal Bifidobacteria, which quickly became the dominant microbiota; their number might make up $60 \%$ to $90 \%$ of all bacteria present in stool samples. Moreover, the colostrum plays a special role in the development of the newborn's intestinal ecosystem. In comparison with mature milk, the colostrum differs primarily in its higher content of protein and vitamin $\mathrm{A}$, but contains less fat and lactose. 
The colostrum is rich in bacteria of the genera Bifidobacterium and Lactobacillus and HMOs. It is also rich in antibodies and immunologic factors including secretory immunoglobulin class A (IgA), cytokines, and anti-inflammatory agents (Dominguez-Bello et al., 2010). Feeding with the colostrum stimulates intestinal colonization by flora and the physiological development of the immune system. In vitro studies comparing the consumption and metabolism of the oligosaccharides present in human milk by the bacteria of Bifidobacterium spp., Escherichia coli, and Clostridium perfringens show that only Bifidobacterium are capable of efficiently metabolizing them and forming lactic acid and short chain fatty acids (Trasande et al., 2013). These metabolites increase the acidity of the intestinal environment, protecting against any invasion of pathogenic bacteria and also stimulating the development of and regenerative capacity of enterocytes.

\section{MECHANISM FOR THE DEVELOPMENT OF METABOLIC DISEASES FROM DISORDERS IN INTESTINAL MICROBIOTA}

An extensive research project, the Human Microbiome Project, whose main goal was to exactly characterize the human microbiota, has provided a wealth of information on the human genome and microbiome (Turnbaugh et al., 2007). Before the completion of the project, it was generally thought that the human genome numbered about 100,000 genes. Surprisingly, about 20,000 protein coding genes were discovered, situating the human genome, in terms of size, next to the genome of Drosophila melanogaster. The human body should be considered as the sum of human cells and microorganisms, and similarly, as the sum of human genes and microorganism genes. The human metabolic phenotype is therefore the result of characteristics encoded in its own genotype and in the genotype of the microbiota inhabiting the body. The microorganisms providing their genetic material give the human body qualities that it would not be able to develop independently. The microbiome of the human body is the source of many functional proteins, including multiple enzymes that specifically affect the functioning of different systems, such as the digestion and absorption of energy from food (Gerber et al., 2015).

\section{THE INCREASED CAPACITY OF ENERGY INTAKE FROM THE DIET IN THE PATHOGENESIS OF OBESITY}

Several studies have found that gut microbiota is an additional factor in the pathophysiology of obesity. The results obtained by Turnbaugh et al. (2007) indicate that the microbiome of obese individuals has an increased capacity to harvest energy from the diet. Analysis of the composition of microbiota from the feces of mice with obesity showed a high content of acetic and butyric acid, as well as a smaller number of calories than in the feces of mice with lean microbiota. Transferring the gut microbiota from obese mice to a normal-weight animal resulted in significantly increased fat content compared to mice with microbiota from lean animals (Turnbaugh et al., 2007).

Some research suggests that the symbiotic microbiota is responsible for $40 \%$ of the total energy intake from food (Koleva et al., 2015). Dysbiosis caused by changes in the composition of gut bacterial species may affect the absorption of energy from food and result in a positive caloric balance in the body. Comparative analyses of the gut microbiota from obese and normal-weight mice show a significant difference in the composition and proportion of bacteria (Cani et al., 2008). In obese individuals, a 50\% decrease in the number of bacteria of the genus Bacteroides and a proportional increase in Firmicutes was observed. In human studies, increases in the number of Bacteroides in the gut were associated with body mass in the obese (Cani et al., 2008). It remains unexplained whether the reduction in the population of Bacteroides is the result of weight gain or is rather the effect of an increase in the intake of nutrients that selectively regulate the growth of these bacteria. It should be emphasized that these effects were observed despite a reduction in the supply of food. Weight gain was accompanied by insulin resistance and other metabolic dysfunctions, such as hypertrophy of the adipocytes and increased levels of blood glucose and leptin. Pathologically altered intestinal microflora promotes the absorption of sugars from the intestinal lumen, resulting in the induction of lipogenesis in the liver (Gerber et al., 2015). Further studies have shown that the process of lipogenesis is actuated by two signaling proteins: ChREBP and SREBP-1. In that study, 
a suppression of the expression of the fasting-induced adipocyte factor was also observed. This factor, often referred to as angiopoietin-like protein 4 , acts as an inhibitor of lipoprotein lipase. The result of its activity is the increased deposition of triglycerides in adipocytes and muscles. Studies on germ-free mice lacking FIAF have shown that they are particularly predisposed to the development of high-calorie diet-induced obesity. These observations also allowed elucidation of the mechanisms responsible for the lack of obesity when the body is fed a typical western diet, rich in sugars and fats, and at the same time devoid of the entire microbial ecosystem. The researchers observed that germ-free mice are protected against diet-induced obesity by two complementary but independent mechanisms that increased fatty acid metabolism. Firstly, the test animals were observed to have an increased concentration of FIAF - a signal to prepare the coactivators for PPAR, which resulted in greater expression of genes encoding proteins that act to regulate fatty acid oxidation in mitochondria. Secondly, there was increased activity of protein kinases dependent on adenosine monophosphate, an enzyme regulating the energy state of the cell. On the basis of these observations, it can be concluded that intestinal microbiota influences the regulation of energy homeostasis of the cell. Intestinal bacteria may affect both sides of the energy balance: on one hand, by adjusting the energy gain from food, and on the other, by affecting the expression of the genes responsible for handling material and energy expenditure (Gerber et al., 2015). The intestinal bacterial microbiota allows more efficient use of dietary carbohydrates by modulating the process of digestion and fat storage in the host. However, it should be emphasized that each type of bacteria has a different metabolic capacity, affecting the extraction of energy from food to varying degrees.

\section{CHRONIC GENERALIZED INFLAMMATION OF MODERATE INTENSITY IN THE PATHOGENESIS OF METABOLIC DISORDERS}

The common denominator of the metabolic diseases, including obesity, is a chronic generalized inflammation of moderate intensity (Blaser and Falkow, 2009; Gerber et al., 2015). For this reason, inflammation is currently cited as the primary mechanism by which metabolic disorders arise. Strains of intestinal bacteria are capable of activating inflammation pathways by interfering with the metabolism of lipids in adipocytes, macrophages, and vascular cells, inducing insulin resistance and the production of trimethylamine oxide (Blaser and Falkow, 2009). These studies highlight the role of the bacterial lipopolysaccharide (LPS) in inducing inflammation combined with metabolic syndrome caused by a high-fat diet. The source of LPS is gram-negative bacteria that live in the intestines. Several experiments on mice have shown that a highfat diet affects the composition of intestinal microflora, promoting the reduction of gram-negative bacteria of the genus Bacteroides and gram-positive bacteria from the groups Eubacteria rectale, Clostridium coccoides, and Bifidobacterium. The ratio of gram-negative bacteria to gram-positive varies considerably in favor of the former (Turnbaugh et al., 2007). These changes in the qualitative and quantitative composition of gut microbiota contribute to a reduction in the integrity of the mucosa and an adverse regrouping of the proteins building tight junctions, such as zonulin- 1 and occludin (Turnbaugh et al., 2007). The study showed a significant decrease in the expression of these proteins, due to high fat levels and the disruption of homeostasis of the intestinal microbiota. This condition is known in the literature as "percolate intestine" and promotes the development of endotoxemia. The transport of LPS into the blood stream from the lumen takes place via chylomicrons, whose number increases considerably with the application of a high-fat diet and pathologically altered tight junctions. One of the results of this is the development of insulin resistance and adipose tissue inflammation caused by adipocyte hypertrophy and the infiltration of macrophages (Blaser and Falkow, 2009). This has also been demonstrated by experiments in mice, in which endotoxemia was induced by continuous subcutaneous infusion of LPS for 4 weeks. Increased blood glucose and insulin level and an increase in body weight and the expression of proinflammatory cytokines were seen in the test animals (Dominguez-Bello et al., 2010). Similarly, experiments involving mice with a mutant CD14 gene on a high-fat diet have shown that endotoxemia intensified the expression of the genes for inflammatory cytokines (activator inhibitor - 1-plasminogen, TNF- $\alpha$, IL-6, and IL-1) through a CD14-dependent 
mechanism. CD14 is a coreceptor of TRL4 located in macrophages, which is critical in the induction of inflammation. This involves bacterial lipopolysaccharide located on the surface of the host immune cells that stimulate the secretion of inflammatory cytokines. The literature indicates that the LPS/CD14 complex also plays a major role in regulating the insulin sensitivity threshold, which contributes significantly to the development of obesity and diabetes. The mechanism behind the creation of these metabolic disorders has been linked to the activation of the intracellular inflammation pathways of the kinases JNK (c-Jun N-terminal kinase) and IKK (IKB kinase; Shoelson et al., 2006). These enzymes are activated by normal inflammatory stimuli, such as LPS, fats, chemokines, and free-fatty acids. The activation of the JNK promotes insulin receptor phosphorylation of IRS-1 (insulin receptor substrate 1) from serine, resulting in impaired signal transduction into the cell and the inhibition of intracellular glucose uptake. The stimulation of the kinase IKK activates a family of nuclear transcription factors NF-kB (nuclear factor), contributing to the development of inflammation and insulin resistance (Shoelson et al., 2006). These reports have been confirmed by studies on humans and laboratory animals. It has been observed that treatment with polymyxin B (which has high efficacy against gram-negative bacteria) lowered the release of LPS in the body and the degree of fatty liver. Trials on patients suffering from type- 2 diabetes showed significantly lower blood levels of bacterial LPS among members of the control group. Antibiotic therapy applied to high-fat fed mice with developed endotoxemia caused a decrease in its intensity through an increase in intestinal integrity, a decrease in levels of inflammatory markers, and an improvement in parameters associated with obesity and diabetes (Jun-Ling and Hui-Ling, 2014).

The literature also points to the endocannabinoid system (eCB) as part of a complex web of activating inflammation in the body that leads to the development of type-2 diabetes. The endocannabinoid system is involved in many physiological processes, including the regulation of energy expenditure, energy metabolism of carbohydrates and lipids, and regulation of the activity of hepatocytes, adipocytes, and endocrine pancreatic function. The $\mathrm{eCB}$ system consists of $\mathrm{CB} 1$ and $\mathrm{CB} 2$ : through the $\mathrm{CB} 1$ receptor, the system controls the permeability of the gut and the plasma levels of LPS. The state of human intestinal microbiota significantly modulates this system (Cani et al., 2008). Studies on obese mice have shown that the supply of prebiotics in the diet results in improvements in the functioning of the intestinal barrier and a reduction in permeability (Shoelson et al., 2006)). Blocking of the CB1 receptor and increased distribution and deployment of zonulin-1 and occludin forming tight junctions has been observed. The studies by Bermudez-Silva et al. on rats showed, however, that activation of the CB2 receptor improves glucose tolerance, and the blocking of the $\mathrm{CB} 1$ receptors demonstrated an effect that mimics the action of $\mathrm{CB} 2$ receptor agonists. The interaction of these receptors significantly modulates the maintenance of glucose homeostasis in the body (Cani et al., 2012). It has been shown that changes in the expression of $\mathrm{CB} 1$ and $\mathrm{CB} 2$ are correlated positively with the quantity of bacteria of the genus $\mathrm{Lac}$ tobacillus and negatively with Clostridium population size (Bermudez-Silva et al., 2007). Experiments conducted on laboratory animals have confirmed that the modulation of the intestinal microflora by the supply of suitable prebiotics has a significant impact on the expression of CB2 receptors (Aguilera et al., 2013). The size of the bacterial population of Akkermansia muciniphila also serves as an indicator of metabolic disorders in humans. Akkermansia muciniphila is an enteric bacterium that degrades mucoproteins of the intestinal mucosa; its presence is negatively correlated with body weight. As research indicates, supplementation with live Akkermansia muciniphila bacteria reduces the severity of the metabolic dysfunction caused by a high-fat diet (Rousseaux et al., 2007). Numerous scientific studies designed to demonstrate the connection between individual strains of bacteria in the intestines and type- 2 diabetes have provided conflicting results. In most cases, researchers have agreed with the reduction in the population of Clostridium and the increase in Betaproteobacteria in patients with type-2 diabetes; the issue that remains concerns whether or not to alter the bacterial population of Firmicutes and of the genera Lactobacillus, Atopobium, and Prevotella. The differences described here are due to a multitude of environmental factors affecting the differentiation of human intestinal microbiota (living environment, type of diet, and research methods) (Ley et al., 2005). 
One of the mechanisms described in the literature on the development of metabolic dysfunctions associated with intestinal dysbiosis is the metabolism of bile acids, which is modulated significantly by bacteria inhabiting the human gut. In the liver, there are two primary synthesized acids: cholic acid and chenodeoxycholic acid. These compounds are converted in the intestines into secondary deoxycholic acid and lithocholic acid. This conversion involves a significant proportion as a group of the intestinal microbiota. Deoxycholic acid is abundant in the bile ducts, and is formed in the large intestine from cholic acid by $7 \alpha$-dehydroxylation catalyzed by certain strains of the genus Clostridium. There is a close relationship between the synthesis of bile acids in the body and the state of the intestinal microbiota. The literature clearly points to the strong antibacterial properties of bile acids which, by destroying cell membranes, reduce bacterial populations (Sato et al., 2014). Only a few microbial communities are able to survive in the intestine, given the high concentrations of bile acids. Interesting results have been obtained from experiments involving rats supplemented with cholic acid. Significant changes were observed in the composition of the intestinal ecosystem on the cluster level. Indicators of bacteria of the phylum Firmicutes and the genus Bacteroidetes increased significantly, indicating a similarity to the changes induced by a high-fat diet. In studies of germ-free mice and mice cultured in the conventional manner, it was observed that the latter were characterized by significantly lower levels of bile in the gall bladder and small intestine and significantly higher in the colon, cecum, serum, and feces. This analysis showed $71 \%$ lower overall levels of bile acids in mice reared under conventional conditions than in germfree animals. The mechanism reducing the synthesis of bile acid in the body is activated by the farnesoid $\mathrm{X}$ nuclear receptor (FXR). Intestinal bacteria are capable of activating this, which results in a reduction in the expression level of the enzyme catalyzing the synthesis of bile acids (Turnbaugh et al., 2006). According to current reports, bile acids play a significant role in the metabolism of glucose, acting as signaling molecules and ligands of cell membrane receptors. They have the ability to activate the FXR nuclear receptor and membrane-bound $G$ protein-coupled receptor (GPCR-1) (Islam et al., 2011). The effects of bile acids have also been demonstrated on the inhibition of gene expression associated with the process of gluconeogenesis, e.g., encoding fructose-1,6-biphosphatase 1 , phosphoenolpyruvate carboxykinase, and glucose6-phosphatase via interaction with the FXR receptor (Turnbaugh et al., 2006). Experiments on mice lacking the FXR receptor have shown that this treatment has a protective effect against the onset of diet-induced obesity and alleviates hyperglycemia, increasing glucose tolerance. In test animals, increased, peripheral glucose uptake and increases in the sensitivity of adipose tissue on the insulin action were observed. Interesting observations have also been provided by a study involving patients with a vertical sleeve gasterectomy (VSG), an effective method for reducing the level of obesity and related pathologies, such as type-2 diabetes. The researchers observed changes in the composition of the intestinal microbiota and the increased circulation of bile acids interacting with the receptor FXR. The results included an improvement in glucose tolerance and the effective maintenance of weight loss. Studies on mice demonstrated that activation of the GPCR-1 receptor located in the enteroendocrine L cells induces the release of glucagonlike peptide (GPL1), improving the functioning of the liver and pancreas and improving glucose tolerance. GPCR-1 receptors are also located in the muscles and brown adipose tissue, and their activation results in increased power consumption and a decrease in the degree of diet-induced obesity (Hylemon et al., 2009). Studies involving patients with type-2 diabetes and impaired glucose tolerance have demonstrated significantly higher levels of taurine-conjugated bile acids, proportional to the severity of the disorder. The lack of adequate research does not allow this parameter to be directly associated with obesity or the consumption of glucose. However, the fact is that the increased levels of taurine-conjugated bile acids that characterize patients with type- 2 diabetes may be associated with the reduced level of the deconjugation process caused by lack of bile acid hydrolase enzyme, an important source of which is the gut microbiota. The compounds that bind bile acids in the body play an important role in preventing the formation of plaque. The binding of bile acids in the intestinal tract stimulates the synthesis of cholesterol in the liver, thereby reducing the level of LDL in serum. The literature also describes 
the significant effect of bile acid sequestrants in the improvement of glucose control in patients with type2 diabetes. The mechanisms leading to this effect include changes in the composition of intestinal microbiota, improvements in the hepatic metabolism of glucose, increases in the release of incretin hormones, and a change in the composition of bile acids (Ryan et al., 2014).

The literature extensively describes the role of the condition of the intestinal microbiota in the pathogenesis of atherosclerosis and dyslipidemia. Recent scientific reports classify the condition of the human intestinal microbiota as one of the environmental factors that stimulate the development of cardiovascular disease (Blaser and Falkow, 2009). Studies using germ-free mice with an experimental model of human atherosclerosis lacking ApoE, a component of the lipoproteins that transport cholesterol in the circulatory system, showed that the lack of intestinal symbiotic microbiota induces plaque, in spite of the low supply of cholesterol from the diet. Colonization of intestinal microbiota typical of the digestive tract prevented the development of atherosclerotic lesions in mice lacking apolipoprotein E with a low supply of cholesterol in the diet. This effect is not achieved with high cholesterol diets, but this does not change the fact that commensal intestinal microbiota has an atheroprotective effect (Jones et al., 2008).

As described in the literature, the mechanisms through which intestinal microbiota affect the development of cardiovascular diseases include phosphatidylcholine metabolism. Intestinal bacteria metabolize choline and phosphatydylocholin to trimethylamine (TMA), which is then converted to a proatherogenic agent, trimethylamine oxide (TMAO). Experiments on germ-free mice lacking ApoE have shown that a diet rich in red meat, a rich source of L-carnitine and trimethylamine, stimulates changes in the composition of intestinal microbiota, whose end result is the production of increased amounts of TMAO and acceleration of atherosclerosis (Stepankova et al., 2010). The variability of the composition of the intestinal microbiota significantly affects the intensity of the production of TMAO in the body, which is a particularly important part of the production of bacteria of the genera Prevotella, Defferibacteres, and Teneriticutes; a lower level of TMAO production is characterized by bacteria of the genus Bacteroides. These results are confirmed by observations in humans using a vegetarian diet and a diet rich in meat. In the case of the vegetarian diet, the treatment group showed significantly lower levels of L-carnitine in the blood, lower production of TMAO in the body and a lower cardiovascular risk. On the other hand, the subjects consuming the diet rich in meat were characterized by the occurrence of cardiovascular events (Wang et al., 2011). Trimethylamine oxide inhibits the reuptake of cholesterol and increases the expression of proatherogenic scavenger receptors (SRS) and SRA CD36 on the surface of macrophages, stimulating them to accumulate cholesterol and to convert themselves into foam cells, an early indicator of atherosclerosis (Wang et al., 2011). Furthermore, this compound reduces bile acid synthesis from cholesterol and the expression of genes encoding proteins for liver bile acid transporters (Wang et al., 2011). In the light of these reports, it can be concluded that a diet high in animal fat and red meat promotes intestinal microbiota dysbiosis which, with the participation of molecules provided in the diet, significantly modifies the metabolism of lipids, thus contributing to an increased risk of cardiovascular disease (Blaser and Falkow, 2009).

Current reports discuss the impact of gut microbiota on the metabolism of anthocyanins, whose sources in the diet are fruits such as blueberries, blackberries, elderberry, and orange vegetables like carrots and sweet potatoes. It is estimated that the process is of significant atheroprotective importance. Protocatechuic acid (PCA) is produced directly by the intestinal microbiota from cyanidin glucoside (Cy-3-G) and has an important antiatherogenic effect, as confirmed by experiments on ApoE knockout mice (Wang et al., 2011). PCA stimulates the outflow of cholesterol from macrophages by activating the expression of sterol transporters ABCA1 and ABCG1. A study by Wang et al. (2012) demonstrated that Cy-3-G improves the level of cholesterol in serum and increases bile acid production by binding directly to the liver $\mathrm{X}$ receptor $(\mathrm{LXR} \alpha)$, activating the expression of hydrolase CYP7A1 (cholesterol 7- $\alpha$-hydrolase). This enzyme is involved in the catabolism of cholesterol into bile acids. Animal studies have shown that Cy-3-G restores the ability to produce nitric oxide in vascular endothelial cells, thereby improving its overall form, 
and also inhibits the process of atherosclerosis. The effect of Cy-3-G on the inhibition of TLR4-dependent inflammatory processes in macrophages was further observed. Cy-3-G stimulates the LXR $\alpha /$ ABCG1 axis, activating the reuptake of cholesterol, thereby lowering its pool in systemic circulation. This dye also reduces the translocation of the TLR4 receptor to the atherosclerotic plaques, thereby preventing its excessive proliferation (Wang et al., 2012).

\section{CONCLUSIONS}

The global epidemic of obesity and other metabolic diseases, in particular of type- 2 diabetes and atherosclerosis, has led to the search for both environmental and internal factors that may have an impact on their development. The fact is that intestinal microbiota has a significant impact on the health of the organism and on the development of such dysfunctions. The complexity of the human body and interactions between metabolic diseases and the gut microbiota means that research is constantly developing in this area. Accurate knowledge of commensal bacteria strains and their impact on the host could become a milestone in the development of "targeted microbial therapy" of metabolic diseases with a proven immune-mediated background.

\section{REFERENCES}

Aguilera, M., Vergara, P., Martínez, V. (2013). Stress and antibiotics alter luminal and wall-adhered microbiota and enhance the local expression of visceral sensory-related systems in mice. Neurogastroent. Motil., 25, 515-529.

Arrieta, M. C., Stiemsma, L. T., Amenyogbe, N., Brown, E. M., Finlay, B. (2014). The intestinal microbiome in early life: health and disease. Front Immunol., 5, 5.

Bermudez-Silva, F. J., Sanchez-Vera, I., Suárez, J., Serrano, A., Fuentes, E., Juan-Pico, ..., Rodríguez de Fonseca, F. (2007). Role of cannabinoid CB2 receptors in glucose homeostasis in rats. Eur. J. Pharmacol., 565, 207-211.

Blaser, M. J., Falkow, S. (2009). What are the consequences of the disappearing human microbiota? Nat. Rev. Micro, 7, 887-894.

Cani, P. D., Bibiloni, R., Knauf, C., Waget, A., Neyrinck, A. M., Delzenne, N. M., Burcelin, R. (2008). Changes in gut microbiota control metabolic endotoxemia-induced inflammation in high-fat diet-induced obesity and diabetes in mice. Diabetes, 57, 1470-1481.

Cani, P. D., Osto, M., Geurts, L., Everard, A. (2012). Involvement of gut microbiota in the development of lowgrade inflammation and type 2 diabetes associated with obesity. Gut Microbes., 3, 279-288.

Clark, S. F., Murphy, E. F., Nilaweera, K., Ross, P. R., Shanahan, F., O'Toole P. W., Cotter, P. D. (2012). The gut microbiota and its relationship to diet and obesity: New insights. Gut Microbes., 3(3), 186-202.

Cox, L. M., Blaser, M. J. (2013). Pathways in microbe-induced obesity Cell Metab., 17, 883-894.

Dietert, R. R., Dietert, J. M. (2015). The Microbiome and sustainable healthcare. Healthcare, 3, 100-129.

Dominguez-Bello, M. G., Costello, E. K., Contreras, M., Magris, M., Hidalgo, G., Fierer, N., Knight, R. (2010). Delivery mode shapes the acquisition and structure of the initial microbiota across multiple body habitats in newborns. Proc. Natl. Acad. Sci. USA, 107, 11971-11975.

Fallani, M., Amarri, S., Uusijarvi, A., Adam, R., Khanna, S., Aguilera, M. (2011). Determinants of the human infant intestinal microbiota after the introduction of first complementary foods in infant samples from five European centres. Microbiology, 157, 1385-1392.

Galdeano, C. M., Perdigo, G. (2006). The probiotic bacterium Lactobacillus casei induces activation of gut mucosal immune system through innate immunity. Clin. Vacc. Immunol., 13, 219-226.

Gerber, J. S., Prasad, P. A., Russell Localio, A., Fiks, A. G., Grundmeier, R. W., Bell, L.M., ..., Zaoutis, T. E. (2015). Variation in antibiotic prescribing across a pediatric primary care network. J. Pediatric. Infect. Dis. Soc., 4(4), 297-304.

Hooper, L. V., Littman, D. R., Macpherson, A. J. (2012). Interactions between the microbiota and the immune system. Science, 336, 1268-1273.

Hylemon, P. B., Zhou, H., Pandak, W. M., Ren, S., Gil, G., Dent, P. (2009). Bile acids as regulatory molecules. J. Lipid Res., 50, 1509-1520.

Islam, K. B., Fukiya, S., Hagio, M., Fujii, N., Ishizuka, S., Ooka, T., ..., Yokota, A. (2011). Bile acid is a host factor that regulates the composition of the cecal microbiota in rats. Gastroenterology, 141, 1773-1781.

Jeurink, P. V., van Bergenhenegouwen, J., Jiménez, E., Knippels, L. M., Fernández, L., Garssen, J., ..., Martín, R. (2013). Human milk: a source of more life than we imagine. Benef. Microbes., 4, 17-30.

Jones, B. V., Begley, M., Hill, C., Gahan, C. G., Marchesi, J. R. (2008). Functional and comparative metagenomic analysis of bile salt hydrolase activity in the human 
gut microbiome. Proc. Natl. Acad. Sci. USA, 105, 13580-13585.

Jun-Ling, H., Hui-Ling, L. (2014). Intestinal microbiota and type 2 diabetes: From mechanism insights to therapeutic perspective. World J. Gastroenterol., 21, 17737-17745.

Koleva, P. T., Kim, J. S., Scott, J. A., Kozyrskyj, A. L. (2015). Microbial programming of health and disease starts during fetal life. Birth Defects Res. C, Embryo Today, 4, 265-277.

Koren, O., Goodrich, J. K., Cullender, T. C., Spor, A., Laitinen, K., Bäckhed, H. K., ..., Ley, R. E. (2012). Host remodeling of the gut microbiome and metabolic changer during pregnancy. Cell, 150, 470-480.

Ley, R. E., Bäckhed, F., Turnbaugh, P., Lozupone, C. A., Knight, R. D., Gordon, J. I. (2005). Obesity alters gut microbial ecology. Proc. Nat. Acad. Sci. USA, 102, 11070-11075.

Lozupone, C. A., Stombaugh, J., Gonzalez, A., Ackermann, G., Wendel, D., Vazquez-Baeza, Y., ..., Knight, R. (2013). Meta-analyses of studies of the human microbiota. Genome Res., 23, 1704-1714.

Mshvildadze, M., Neu, J., Shuster, J., Theriaque, D., Li, N., Mai, V. (2010). Intestinal microbial ecology in premature infants assessed with non-culture-based techniques. J. Pediatr., 156(1), 20-25.

Penders, J., Thijs, C., Vink, C. (2006). Factor influencing the composition of the intestinal microbiota in early infancy. Pediatrics, 118(2), 511-521.

Ridaura, V. K., Faith, J. J., Rey, F. E., Cheng, J., Duncan, A. E., Kau, A. L., ..., Gordon, J. I. (2013). Gut microbiota from twins discordant for obesity modulate metabolism in mice. Science, 6, 341(6150), 12412-12414.

Rousseaux, C., Thuru, X., Gelot, A., Barnich, N., Neut, C., Dubuquoy, L., ..., Chamaillard, M. (2007). Lactobacillus acidophilus modulates intestinal pain and induces opioid and cannabinoid receptors. Nat. Med., 13, 35-37.

Ryan, K. K., Tremaroli, V., Clemmensen, C., Kovatcheva-Datchary, P., Myronovych, A., Karns, R., ..., Bäckhed, F. (2014). FXR is a molecular target for the effects of vertical sleeve gastrectomy. Nature, 509, 183-188.

Sato, J., Kanazawa, A., Ikeda, F., Yoshihara, T., Goto, H., Abe, H., ..., Ogihara, T. (2014). Gut dysbiosis and detection of "live gut bacteria" in blood of Japanese patients with type 2 diabetes. Diabetes Care, 37, 2343-2350.
Shoelson, S. E., Lee, J., Goldfine, A. B. (2006). Inflammation and insulin resistance J. Clin. Invest., 116, 1793-1801.

Stepankova, R., Tonar, Z., Bartova, J., Nedorost, L., Rossman, P., Poledne, R. (2010). Absence of microbiota (germ-free conditions) accelerates the atherosclerosis in ApoE-deficient mice fed standard low cholesterol diet. J. Atheroscler. Thromb., 17(8), 796-804.

Trasande, L., Blustein, J., Liu, M., Corwin, E., Cox, L. M., Blaser, M. J. (2013). Infant antibiotic exposures and early-life body mass. Int. J. Obes., 37, 16-23.

Tsakok, T., McKeever, T. M., Yeo, L., Flohr, C. (2013). Does early life exposure to antibiotics increase the risk of eczema? A systematic review. Br. J. Dermatol., 169, 983-991.

Turnbaugh, P. J., Hamady, M., Yatsunenko, T., Cantarel, B. L., Duncan, A., Ley, R. E., ..., Gordon, J. I. (2009). A core gut microbiome in obese and lean twins. Nature, 457, 480-484.

Turnbaugh, P. J., Ley, R. E., Hamady, M. (2007). The human microbiome project. Nature, 449, 804-810.

Turnbaugh, P. J., Ley, R. E., Mahowald, M. A., Magrini, V., Mardis, E. R., Gordon, J. I. (2006). An obesity-associated gut microbiome with incrased capacity for energy harvest. Nature, 444(7122), 1027-1031.

Turnbaugh, J. P., Ley, R. E., Hamady, M., Fraser-Liggett, C., Knight, R., Gordon, J. I. (2007). The human microbiome project: exploring the microbial part of ourselves in a changing world. Nature, 18, 804-810.

Wang, Z., Klipfell, E., Bennett, B. J., Koeth, R., Levison, B. S., Dugar, B., ..., Hazen, S. L. (2011). Gut flora metabolism of phosphatidylcholine promotes cardiovascular disease. Nature, 472(7341), 57-63.

Wang, D., Zou, T., Yang, Y., Yan, X., Ling, W. (2011). Cyanidin-3-O- $\beta$-glucoside with the aid of its metabolite protocatechuic acid, reduces monocyte infiltration in apolipoprotein E-deficient mice. Biochem. Pharmacol., 82(7), 713-719.

Wang, D., Xia, M., Yan, X., Li, D., Wang, L., Xu, Y., Jin, T., Ling, W. (2012). Gut microbiota metabolism of anthocyanin promotes reverse cholesterol transport in mice via repressing miRNA-10b. Circ. Res., 111(8), 967-981. 